Check for updates

Cite this: RSC Adv., 2017, 7, 33600

\title{
Effect of flake size on the mechanical properties of graphene aerogels prepared by freeze casting $\dagger$
}

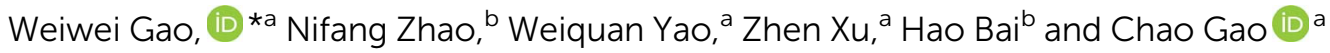

Aerogels enable a wide range of potential applications owing to their ultralow density, superelasticity, high specific surface area, energy-absorbability and so on. However, it is usually difficult to precisely control their mechanical performance, which largely hinders their applications. Here, we prepared anisotropic graphene aerogels assembled with flakes having different sizes ranging from sub-micron to $\sim 80 \mu \mathrm{m}$ by the freeze casting technique, using ice as a template to assembling graphene oxide (GO) sheets into 3-dimensional (3D) aerogels. We found that graphene flake size has a profound effect on the mechanical performance of the assembled graphene aerogels, particularly their strength, modulus and fatigue resistance under compression. Larger flakes had stronger interaction when assembled, which made them more resistant to slipping between adjacent flakes during deformation. As a result, the graphene aerogel with larger flake size showed both higher strength and fatigue resistance. Our research provides a new way of controlling the mechanical properties of graphene aerogel by only adjusting the intrinsic properties of the flakes, e.g., size, without crosslinking agent or co-assembly with other materials as in previous studies.
\end{abstract}

Received 17th May 2017

Accepted 29th June 2017

DOI: $10.1039 / \mathrm{c} 7 \mathrm{ra05557a}$

rsc.li/rsc-advances aspect ratio are used. Although some progresses have been made in this direction, there is still no systematic study linking the properties of the building block (graphene oxide flake) and its assembly (graphene aerogel), which requires tailoring architecture at multiple scales.

Here, we present the effect of flake size on mechanical properties of graphene aerogel. A series of elastomeric graphene aerogels with flakes having a wide range of size, from submicron to $\sim 80 \mu \mathrm{m}$, were prepared by the freeze casting technique. It showed that the graphene flake size has a profound effect on the mechanical performance of the assembled graphene aerogels, particularly their strength, modulus and fatigue resistance under compression. Larger flakes had stronger interaction with adjacent ones when assembled, making them more resistant to slip during deformation. As a result, the graphene aerogel assembled by larger flake size showed both higher strength and fatigue resistance. Different from previous efforts in tailoring graphene aerogels' mechanical properties where crosslinking agents and co-assembly with other materials were usually employed, we controlled their properties by only the flake size. By adjusting the intrinsic properties of the flakes, particularly their size, our research provides a new way of controlling the mechanical properties of graphene aerogel, which is crucial for its multifunctionality and applications.

\section{Materials and methods}

\section{Materials}

Graphene oxide (GO) suspension with $\sim 80 \mu \mathrm{m}$ in size was received from Hangzhou Gaoxi Technology Co. Ltd. (http:// 
www.gaoxitech.com) (Zhejiang, China). Aqueous Poly (vinyl alcohol) (PVA, Mw = 20 500, 99\%) was purchased from Aladdin Chemistry Co., Ltd, China.

\section{Preparation of graphene oxide precursor suspension}

GO with $\sim 80 \mu \mathrm{m}$ in size was sonicated for different amount of time with high energy to break down. After sonication for 3, 5, and 20 minutes, the GO flake size was reduced to $\sim 50 \mu \mathrm{m}, \sim 20$ $\mu \mathrm{m}$, and $\sim 1 \mu \mathrm{m}$, respectively. The concentrated GO suspensions of $10 \mathrm{mg} \mathrm{ml}^{-1}$ were obtained by centrifugation. PVA solution (10 $\mathrm{mg} \mathrm{ml}^{-1}$ ) was added to the GO suspension. The ratio of GO : PVA was kept at $1: 1$ in weight.

\section{Preparation of graphene oxide aerogels}

Graphene oxide aerogels were fabricated by the unidirectional freeze casting method. The precursor suspension (GO : PVA = $5: 5 \mathrm{mg} \mathrm{ml}^{-1}$ ) was poured into a square tube and then frozen at three typical temperatures of $-196{ }^{\circ} \mathrm{C}$ (Liquid Nitrogen, LN), $-90{ }^{\circ} \mathrm{C}$, and $-50{ }^{\circ} \mathrm{C}$. After the precursor suspension was frozen entirely, the sample was tapped out of the mold and freezedried for more than $48 \mathrm{~h}$ at $-80{ }^{\circ} \mathrm{C}$ with Freeze dryer under 0.05 mbar pressure (Labconco 8811, Kansas City, USA). Finally, the graphene aerogels were reduced by thermal treatment at $800{ }^{\circ} \mathrm{C}$ for $2 \mathrm{~h}$ in a $5 \% \mathrm{H}_{2} / 95 \% \mathrm{Ar}$ mixed gas.

\section{Characterizations}

GO flake size was checked using over 150 flakes by scanning electron microscopy (SEM) images, obtained by Hitachi S-3700 at an acceleration voltage of $10 \mathrm{kV}$ in secondary electron mode. SEM samples were prepared by drop casting of diluted GO suspension on the silicon substrates. Mechanical properties of the prepared graphene aerogels were investigated in the compressive/tensile mode by an electronic universal testing machine (UTM2102, ShenZhen Suns Technology Stock Co., Ltd, China).

\section{Results and discussion}

Fig. 1a-d shows SEM images of graphene oxide (GO) flakes with different sizes. The small GO was fabricated by sonicating commercial large GO flake. The flake size was properly controlled by the sonication power and time. ${ }^{6}$ SEM images show that large GO flakes were successfully broken down by our sonication method. The initial flake size was $\sim 80 \mu \mathrm{m}$, and reduced to $\sim 50 \mu \mathrm{m}, \sim 20 \mu \mathrm{m}$ and $\sim 1 \mu \mathrm{m}$ after proper treatment, which are defined as GO-1 $(\sim 80 \mu \mathrm{m})$, GO-2 $(\sim 50 \mu \mathrm{m})$, GO-3 $(\sim 20$ $\mu \mathrm{m})$, and GO-4 $(\sim 1 \mu \mathrm{m})$, respectively. The GO flake size was measured by SEM technique. The size distribution of the treated GO flakes was summarized respectively in Fig. 1e-h, based on the SEM image analysis of over 150 flakes with more than 5 different locations. Fig. 1i-l show the optical images of graphene aerogel assembled with different sized GO flakes. All the aerogels look similar in appearance.

The fabrication route of graphene aerogels with different flake sizes was illustrated in Fig. 2. ${ }^{3,34}$ Firstly, the water suspension with GO flakes were prepared by dispersing and

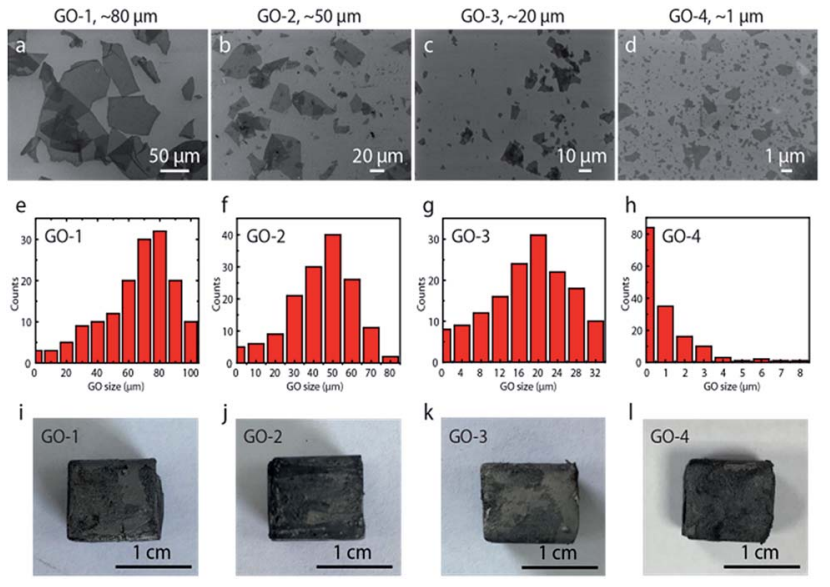

Fig. 1 Graphene oxide (GO) flakes with different size. (a-d) SEM images of GO flakes with the size of $\sim 80 \mu \mathrm{m}$ for GO-1 (a), $\sim 50 \mu \mathrm{m}$ for GO-2 (b), $\sim 20 \mu \mathrm{m}$ for GO-3 (c), and $\sim 1 \mu \mathrm{m}$ for GO-4 (d), respectively. $(e-h)$ Show the corresponding size distribution of GO flakes in $(a-d)$. The smaller flakes (GO-2, GO-3, and GO-4) were made by sonicating GO-1. The flake size was properly controlled by sonication power and time. (i-l) Optical images showing the graphene aerogels assembled with different sized GO flakes, which all look similar in appearance.

dissolving GO and PVA into water. The ratio of GO : PVA was kept as $1: 1$ in weight $\left(5: 5 \mathrm{mg} \mathrm{ml}^{-1}\right)$. PVA was used to adjust the suspension viscosity for freeze casting. ${ }^{35,36}$ Previous studies have shown that viscosity is a crucial parameter in freeze casting affecting the architecture and properties of the final graphene aerogel. ${ }^{37}$ Secondly, the prepared GO suspensions with different flake size were cast into square Teflon molds placed on the copper rod which is the cold source. The suspension was unidirectionally frozen by decreasing the temperature of the copper rod at a controlled cooling rate. When completely
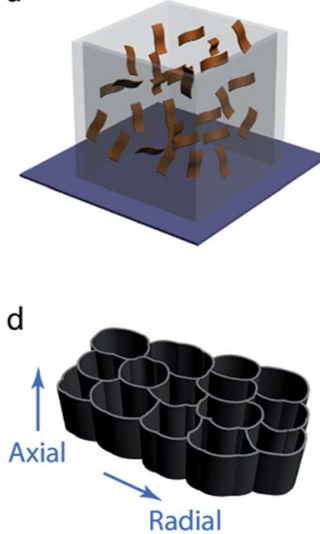

b

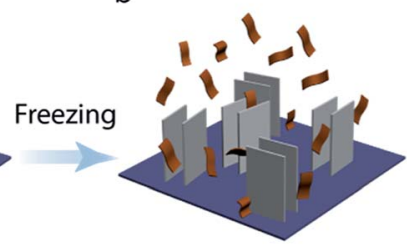

Dry

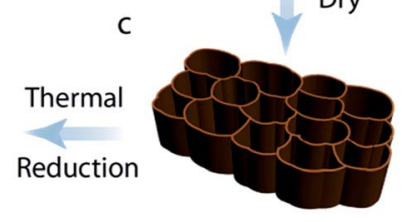

Fig. 2 Fabrication process of graphene aerogel. (a) A GO suspension was firstly prepared by mixing GO flakes with PVA/water solution to control viscosity. (b) During freezing from the cold substrate, the ice crystals grew preferentially from the bottom to the top, expelling the GO flakes in between the ice crystals. (c) Once the suspension was completely frozen, ice was removed by sublimation, leaving a porous scaffold assembled with GO flakes. (d) Anisotropic graphene aerogel was finally realized by thermal reduction of dried GO scaffold. 
frozen, the samples were tapped out of the mold and freezedried for more than 48 hours at $-90{ }^{\circ} \mathrm{C}$ with $1 \mathrm{~Pa}$ pressure (Freeze Dryer 8, Labconco, USA). Reduced anisotropic graphene oxide aerogels were realized by thermal treatment ( 2 hours at $800{ }^{\circ} \mathrm{C}$ ) in a $5 \% \mathrm{H}_{2} / 95 \%$ Ar atmosphere in a tubular oven.

Fig. 3 shows the typical microstructure of graphene oxide aerogel with GO flake size ranging from sub-micron to $\sim 80 \mu \mathrm{m}$. Three typical freezing temperature of $\mathrm{LN},-90{ }^{\circ} \mathrm{C}$, and $-50{ }^{\circ} \mathrm{C}$ were chosen for each GO flake size to systematically investigate the temperature effect on the formation of porous structure. During freeze casting process, ice crystals grow in aqueous phase when graphene oxide flakes are ejected from the moving ice front and aligned between the ice crystals, leading to the formation of continuous 3D network. ${ }^{\mathbf{4}, 6,31}$ Subsequently, the ice crystal is sublimated after freeze drying, leaving a stable freestanding 3D network. The freezing speed usually manipulated the microstructure of graphene aerogel,,$^{33,34,38-40}$ with lower speed resulting in aerogel with larger pore size. Besides, the suspension viscosity also has strong effect on the microstructure: higher viscosity usually leads to smaller pores in the final porous structure. In order to investigate the effect of GO flake size on the mechanical performance, PVA solution was added into all the GO suspensions to improve the viscosity to ensure coherent micro-porous structure of all the prepared graphene aerogels. In Fig. 3, a similar lamellar structure with a honeycomb-like morphology was observed in the aerogels, which is consistent with previously reported carbon based aerogels fabricated by freeze drying. ${ }^{\mathbf{4 , 2 1}}$ As decreasing the freezing temperature from $-50{ }^{\circ} \mathrm{C}$ to $\mathrm{LN}$, the pores became slightly smaller. For example, from $\sim 22 \mu \mathrm{m}$ of $-50{ }^{\circ} \mathrm{C}$ to $\sim 13 \mu \mathrm{m}$ of $\mathrm{LN}$

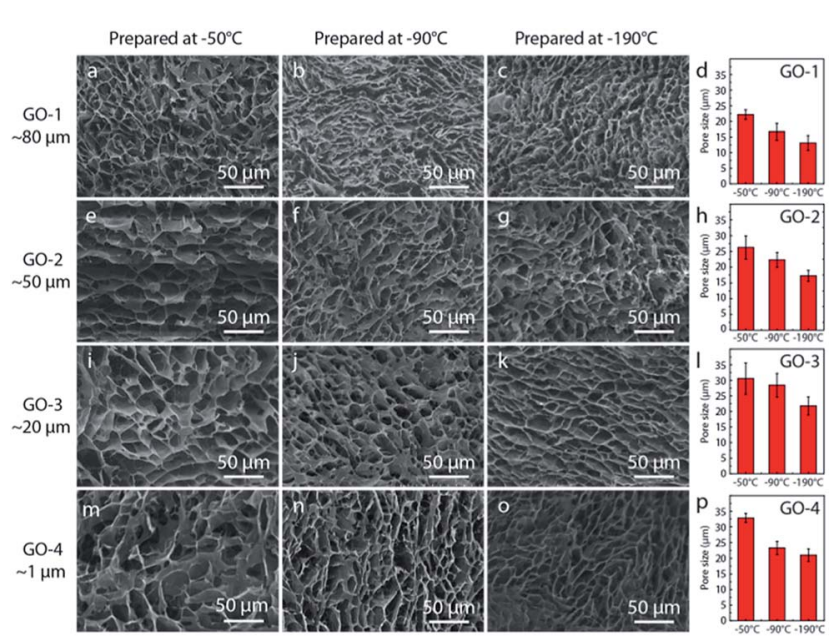

Fig. 3 SEM images characterizing the morphology of graphene aerogels assembled with different GO flake size. Top view of graphene oxide aerogel fabricated by freeze casting of $\mathrm{GO}$ suspension with different flake sizes of $\sim 80 \mu \mathrm{m}$ for GO-1 (a-c), $\sim 50 \mu \mathrm{m}$ for GO-2 (eg), $\sim 20 \mu \mathrm{m}$ for GO-3 (i-k), and $\sim 1 \mu \mathrm{m}$ for GO-4 (m-o), respectively. Three typical freezing temperatures $\left(\mathrm{LN},-90^{\circ} \mathrm{C}\right.$, and $\left.-50{ }^{\circ} \mathrm{C}\right)$ were chosen for each GO flake size. The aerogels exhibit a similar microstructure with a honeycomb-like morphology except that of GO flake with submicron. Pore size distribution of GO aerogel with different freezing temperature and GO flake size shown in $(d, h, l, p)$. The pore size of GO aerogel reduced when decreasing freezing temperature. for aerogel with largest flakes (GO-1) with highest viscosity. Besides, few change in pore size for the aerogels assembled with different sized GO flake, only $\sim 10 \mu \mathrm{m}$ difference. By increasing the viscosity of all the GO suspensions, we controlled the final microstructure of all the prepared graphene aerogels. By minimizing the effect of microstructure, ${ }^{4}$ we were thus able to compare systematically the effect of flake size on the mechanical properties of the graphene aerogels.

Fig. 4 systematically investigated the mechanical properties of the anisotropic graphene aerogels assembled with different sized GO flakes. Three freezing temperatures $\left(\mathrm{LN},-90{ }^{\circ} \mathrm{C}\right.$, and $-50{ }^{\circ} \mathrm{C}$ ) were selected to manipulate the microstructure of the graphene aerogels. It was found that GO flake size had a profound effect on the mechanical properties. In the radial and axial directions of graphene aerogels, both strength and modulus decrease obviously when reducing the GO flake size. Meanwhile, strength values in the axial direction are higher than those of radial direction. For example, at the freezing temperature of $-50{ }^{\circ} \mathrm{C}$, the aerogels assembled with GO-1 (flake size: $\sim 80 \mu \mathrm{m})$, measured in the axial direction, exhibited a maximal strength $(\sim 38 \mathrm{kPa})$ and modulus $(\sim 34 \mathrm{kPa})$ at the strain of $80 \%$, about 8 (strength) and 3 (modulus) times of that assembled with GO-4 (flake size: $\sim 1 \mu \mathrm{m}$ ). In the radial direction, the maximal strength and modulus (GO-1) are $\sim 29 \mathrm{kPa}$ and $\sim 58$ $\mathrm{kPa}$, about 6 (strength) and 24 (modulus) times of graphene aerogels (GO-4). Moreover, graphene aerogels in the radial direction show better compression recovery behaviour, they can fully recover from large strain. For the graphene aerogels assembled with the same flake size but at different freezing temperature, the difference in mechanical properties is
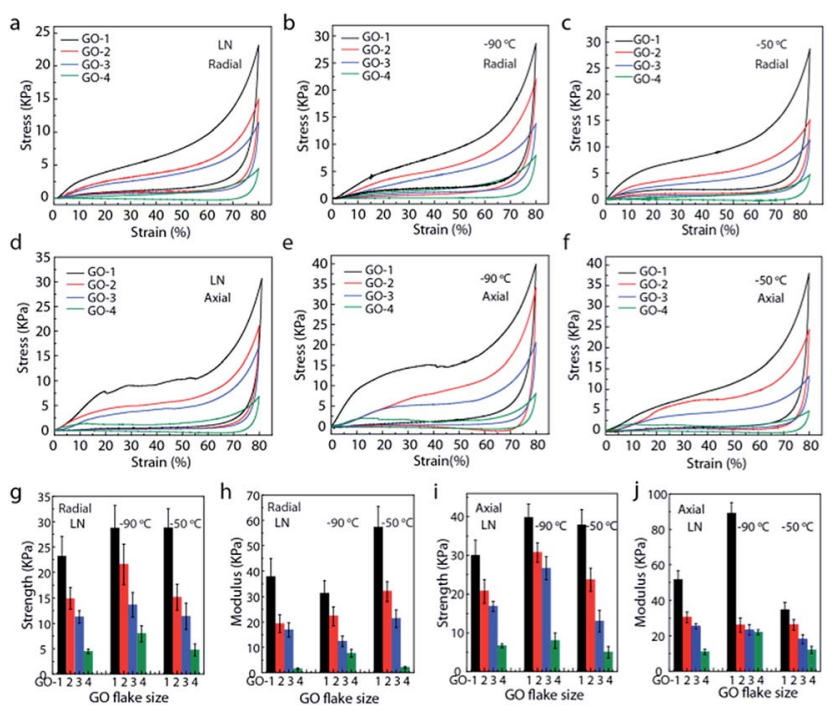

Fig. 4 Mechanical properties of anisotropic graphene aerogels. Compressive stress-strain curves in the radial and axial directions of graphene aerogels assembled with different GO flake size at freezing temperatures of $\mathrm{LN}(\mathrm{a}$ and $\mathrm{d}),-90^{\circ} \mathrm{C}$ (b and e), and $-50^{\circ} \mathrm{C}$ (c and f). Summarized strength ( $g$ and $i$ ) and modulus ( $h$ and $j$ ) of graphene aerogels. GO flake has a profound effect on the mechanical properties of graphene aerogels. Obvious decrease in both strength and modulus were observed when reducing the GO flake size. 
negligible, which could be attributed to their similar microstructure. ${ }^{4,6,31}$ Besides, the stretch properties of anisotropic graphene aerogels were studied in Fig. S1. $\dagger$ Both strength and modulus in axial direction are higher than those of radial direction in all the aerogels. However, aerogels in radial direction possess larger elongation at break.

In order to investigate the fatigue resistance, the cyclic compression recovery performance of graphene aerogels was studied. ${ }^{32}$ Fig. 5 shows the cyclic stress-strain curves of graphene aerogels with different $\mathrm{GO}$ flake sizes at freezing temperature of $\mathrm{LN},-90{ }^{\circ} \mathrm{C}$, and $-50{ }^{\circ} \mathrm{C}$, respectively. With $50 \%$ strain, the aerogels assembled with large GO-1 flakes only experience a slight reduction in height and still remain over $92 \%$ of maximal stress after 100 compression cycles. In contrast, the aerogels with small GO flakes, especially GO-4 (sub-micron) flakes, show relatively poor mechanical performance including lower strength and modulus. Even under applied $50 \%$ strain, the aerogel can only recover to $80 \%$ of its original height and just leave $80 \%$ of stress after 100 compression cycles.

Fig. 6 shows the GO flake deformation mechanism of the aerogel wall composed of flakes with different size during compression. According to the SEM results, our graphene aerogels assembled with different sized GO flakes exhibited similar microstructure and inner morphology, with a narrow pore size distribution limited to $10 \mu \mathrm{m}$. Besides, all the aerogels looked similar in appearance in Fig. 1i-l. In principle, these aerogels should exhibit similar mechanical properties based on the previous reports. ${ }^{4,6,31}$ However, significant difference in mechanical properties were found among our aerogels. Aerogel assembled with large GO flakes had excellent mechanical properties, including higher compressive strength and modulus, as well as good anti-fatigue property during cyclic compression. Based on our study, micro-defects from the pore wall assembled with GO flakes contributed to

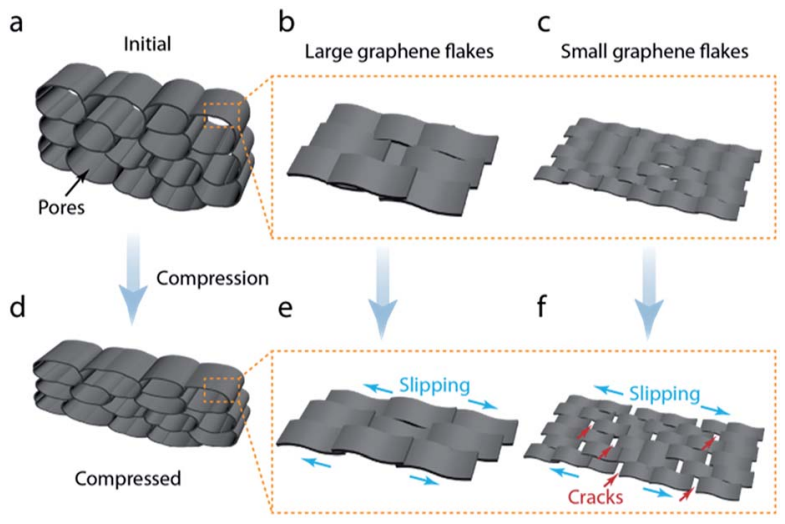

Fig. 6 Schematics showing deformation mechanisms of a single aerogel wall composed of flakes with different size during compression. $(a-c)$ Larger graphene flakes has stronger interaction with adjacent flakes to resist against deformation, usually resulting in 'slipping'. Besides 'slipping', (d-f) smaller flakes are easy to crack as they have weaker inter-flake conjunction.

such significant difference in mechanical performance. Following our special procedure, two separated aerogels with the same microstructure were assembled with large and small GO flakes, respectively. The pore wall constructed by large GO, especially ultra-large size GO flakes, has less defects. Large flakes display stronger interaction with adjacent ones to resist against deformation, usually resulting in 'slipping'. In contrast, the assembly with small size GO usually takes more flakes than that of large flakes, and thus more overlap joints of GO flakes in pore wall, which are easy to crack as they have weaker inter-flake conjunctions during deformation. Our study shows how the intrinsic properties of the building blocks, i.e., GO flake size, could affect the mechanical properties of the macroscopic 3D assembly, i.e., graphene aerogel.

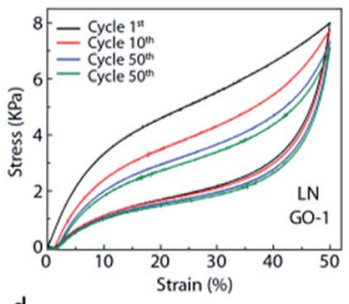

d

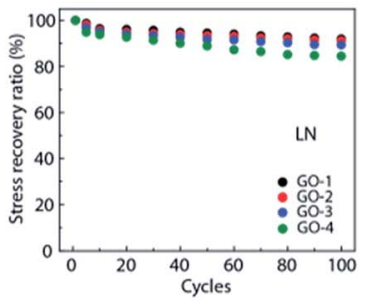

b

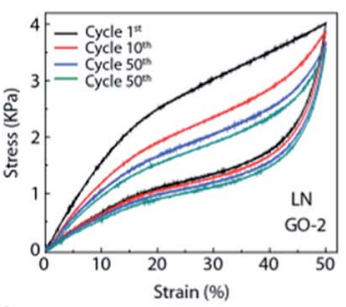

e

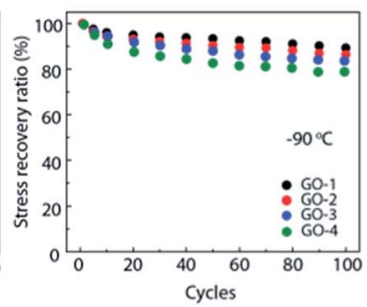

C

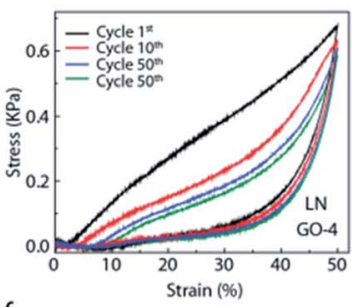

f

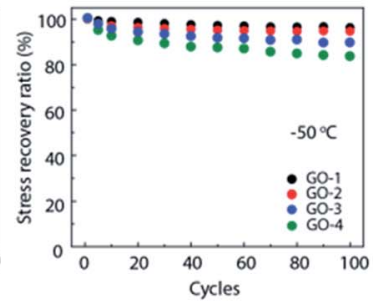

Fig. 5 Fatigue resistance of graphene aerogels. (a-c) Stress-strain curves of graphene aerogels assembled with GO-1 (a), GO-2 (b), and GO-4 (c) flakes at $L N$. (d-f) Summarized stress recovery ratio of graphene aerogels assembled with different sized flakes at $L N(d),-90{ }^{\circ} \mathrm{C}$ (e), and $-50{ }^{\circ} \mathrm{C}(\mathrm{f})$. The graphene aerogel with larger flake size exhibited both higher strength and fatigue resistance. 


\section{Conclusion}

In conclusion, we have prepared a series of anisotropic graphene aerogels with different GO flake size ranging from submicron to $\sim 80 \mu \mathrm{m}$ by the freeze casting technique, using ice as a template in assembling GO flakes into 3D aerogels. We increased the suspension viscosity to generate similar microstructures in the final graphene aerogels, which made it possible to minimize the effect of microstructure on the aerogels' mechanical properties. The aerogels with similar microstructure should show similar mechanical properties as predicted in literature. However, we found that GO flake size, in addition to the microstructure, also has a profound effect on the mechanical performance of the assembled graphene aerogels, particularly their strength, modulus and fatigue resistance under compression. Large flakes had stronger interaction when assembled, which made it more resistant to slip between adjacent flakes during deformation. As a result, the graphene aerogel with larger flake size showed both higher strength and fatigue resistance. By adjusting the intrinsic properties of the flakes, specifically their size, our research provides a new way of controlling the mechanical properties of graphene aerogel, which is crucial for its multifunctional applications.

\section{Acknowledgements}

This work was supported by the National Natural Science Foundation of China (No. 51603183, 21325417, 51533008, 51603182), the Fundamental Research Funds for the Central Universities (No. 2017QNA4036, 2017XZZX008-006), and National Key R\&D Program of China (Grant No. 2016YFA0200200). The '1000 Youth Talents Plan' of China.

\section{References}

1 H. Sun, Z. Xu and C. Gao, Adv. Mater., 2013, 25, 2554-2560.

2 Z. Li, Z. Liu, H. Sun and C. Gao, Chem. Rev., 2015, 115, 70467117.

3 K. Shehzad, Y. Xu, C. Gao and X. Duan, Chem. Soc. Rev., 2016, 45, 5541-5588.

4 L. Qiu, J. Z. Liu, S. L. Chang, Y. Wu and D. Li, Nat. Commun., 2012, 3, 1241.

5 Y. Wu, N. Yi, L. Huang, T. Zhang, S. Fang, H. Chang, N. Li, J. Oh, J. A. Lee, M. Kozlov, A. C. Chipara, H. Terrones, P. Xiao, G. Long, Y. Huang, F. Zhang, L. Zhang, X. Lepro, C. Haines, M. D. Lima, N. P. Lopez, L. P. Rajukumar, A. L. Elias, S. Feng, S. J. Kim, N. T. Narayanan, P. M. Ajayan, M. Terrones, A. Aliev, P. Chu, Z. Zhang, R. H. Baughman and Y. Chen, Nat. Commun., 2015, 6, 6141. 6 N. Ni, S. Barg, E. Garcia-Tunon, F. Macul Perez, M. Miranda, C. Lu, C. Mattevi and E. Saiz, Sci. Rep., 2015, 5, 13712.

7 Z. Lei, Y. Yan, J. Feng, J. Wu, G. Huang, X. Li, W. Xing and L. Zhao, RSC Adv., 2015, 5, 25650-25656.

8 S. Liao, T. Zhai and H. Xia, J. Mater. Chem. A, 2016, 4, 10681077.

9 X. Zhang, P. Liu, Y. Duan, M. Jiang and J. Zhang, RSC Adv., 2017, 7, 16467-16473.
10 M. Yu, W. Qiu, F. Wang, T. Zhai, P. Fang, X. Lu and Y. Tong, J. Mater. Chem. A, 2015, 3, 15792-15823.

11 H. Chen, F. Guo, Y. Liu, T. Huang, B. Zheng, N. Ananth, Z. Xu, W. Gao and C. Gao, Adv. Mater., 2017, 27, 1605958.

12 H. Chang, J. Qin, P. Xiao, Y. Yang, T. Zhang, Y. Ma, Y. Huang and Y. Chen, Adv. Mater., 2016, 28, 3504-3509.

13 Y. Wu, M. Gong, M.-C. Lin, C. Yuan, M. Angell, L. Huang, D.-Y. Wang, X. Zhang, J. Yang, B.-J. Hwang and H. Dai, Adv. Mater., 2016, 28, 9218-9222.

14 H. Bi, X. Xie, K. Yin, Y. Zhou, S. Wan, L. He, F. Xu, F. Banhart, L. Sun and R. S. Ruoff, Adv. Funct. Mater., 2012, 22, 44214425.

15 S. Wan, J. Peng, L. Jiang and Q. Cheng, Adv. Mater., 2016, 28, 7862-7898.

16 Y. Zhang, S. Gong, Q. Zhang, P. Ming, S. Wan, J. Peng, L. Jiang and Q. Cheng, Chem. Soc. Rev., 2016, 45, 2378-2395.

17 K. Chen, X. Tang, Y. Yue, H. Zhao and L. Guo, ACS Nano, 2016, 10, 4816-4827.

18 X. Gui, J. Wei, K. Wang, A. Cao, H. Zhu, Y. Jia, Q. Shu and D. Wu, Adv. Mater., 2010, 22, 617-621.

19 Q. Peng, Y. Li, X. He, X. Gui, Y. Shang, C. Wang, C. Wang, W. Zhao, S. Du, E. Shi, P. Li, D. Wu and A. Cao, Adv. Mater., 2014, 26, 3241-3247.

20 T. Zhang, H. Chang, Y. Wu, P. Xiao, N. Yi, Y. Lu, Y. Ma, Y. Huang, K. Zhao, X.-Q. Yan, Z.-B. Liu, J.-G. Tian and Y. Chen, Nat. Photonics, 2015, 9, 471-476.

21 X. Xu, Q. Zhang, Y. Yu, W. Chen, H. Hu and H. Li, Adv. Mater., 2016, 28, 9223-9230.

22 C. Bao, S. Bi, H. Zhang, J. Zhao, P. Wang, C. Y. Yue and J. Yang, J. Mater. Chem. A, 2016, 4, 9437-9446.

$23 \mathrm{H}$. Hu, Z. Zhao, W. Wan, Y. Gogotsi and J. Qiu, Adv. Mater., 2013, 25, 2219-2223.

24 L. Qiu, D. Liu, Y. Wang, C. Cheng, K. Zhou, J. Ding, V. T. Truong and D. Li, Adv. Mater., 2014, 26, 3333-3337.

25 L. Qiu, M. Bulut Coskun, Y. Tang, J. Z. Liu, T. Alan, J. Ding, V.-T. Truong and D. Li, Adv. Mater., 2016, 28, 194-200.

26 Z. Chen, W. Ren, L. Gao, B. Liu, S. Pei and H.-M. Cheng, Nat. Mater., 2011, 10, 424-428.

27 M. A. Worsley, P. J. Pauzauskie, T. Y. Olson, J. Biener, J. H. Satcher and T. F. Baumann, J. Am. Chem. Soc., 2010, 132, 14067-14069.

28 Y. Li, J. Chen, L. Huang, C. Li, J.-D. Hong and G. Shi, Adv. Mater., 2014, 26, 4789-4793.

29 H. Hu, Z. Zhao, W. Wan, Y. Gogotsi and J. Qiu, Adv. Mater., 2013, 25, 2219-2223.

30 Y. Xu, K. Sheng, C. Li and G. Shi, ACS Nano, 2010, 4, 43244330.

31 S. Barg, F. M. Perez, N. Ni, P. do Vale Pereira, R. C. Maher, E. Garcia-Tuñon, S. Eslava, S. Agnoli, C. Mattevi and E. Saiz, Nat. Commun., 2014, 5, 4328.

32 H. L. Gao, Y. B. Zhu, L. B. Mao, F. C. Wang, X. S. Luo, Y. Y. Liu, Y. Lu, Z. Pan, J. Ge, W. Shen, Y. R. Zheng, L. Xu, L. J. Wang, W. H. Xu, H. A. Wu and S. H. Yu, Nat. Commun., 2016, 7, 12920.

33 S. Deville, E. Saiz, R. K. Nalla and A. P. Tomsia, Science, 2006, 311, 515-518. 
34 H. Bai, Y. Chen, B. Delattre, A. P. Tomsia and R. O. Ritchie, Sci. Adv., 2015, 1, e1500849.

35 H. Bai, C. Li, X. Wang and G. Shi, Chem. Commun., 2010, 46, 2376-2378.

36 R. Verdejo, M. M. Bernal, L. J. Romasanta and M. A. LopezManchado, J. Mater. Chem., 2011, 21, 3301-3310.

37 C. Zhu, T. Y.-J. Han, E. B. Duoss, A. M. Golobic, J. D. Kuntz, C. M. Spadaccini and M. A. Worsley, Nat. Commun., 2015, 6, 6962.
38 H. Bai, F. Walsh, B. Gludovatz, B. Delattre, C. Huang, Y. Chen, A. P. Tomsia and R. O. Ritchie, Adv. Mater., 2016, 28, 50-56.

39 N. Zhao, M. Yang, Q. Zhao, W. Gao, T. Xie and H. Bai, ACS Nano, 2017, 11, 4777-4784.

40 M. Yang, N. Zhao, Y. Cui, W. Gao, Q. Zhao, C. Gao, H. Bai and T. Xie, ACS Nano, 2017, DOI: 10.1021/acsnano.7b01815. 